\title{
Deterministic Bipolar Compressed Sensing Matrices from Binary Sequence Family
}

\author{
Cunbo $\mathrm{Lu}^{1}$, Wengu Chen ${ }^{1 *}$ and Haibo $\mathrm{Xu}^{1}$ \\ ${ }^{1}$ Institute of Applied Physics and Computational Mathematics \\ Beijing, 100088 - China \\ [e-mail: 444180647@qq.com, chenwg@iapcm.ac.cn, xu_haibo@iapcm.ac.cn] \\ *Corresponding author: Wengu Chen
}

Received September 25, 2019; revised November 1, 2019; revised March 16, 2020; accepted April 1, 2020; published June 30, 2020

\begin{abstract}
For compressed sensing (CS) applications, it is significant to construct deterministic measurement matrices with good practical features, including good sensing performance, low memory cost, low computational complexity and easy hardware implementation. In this paper, a deterministic construction method of bipolar measurement matrices is presented based on binary sequence family (BSF). This method is of interest to be applied for sparse signal restore and image block CS. Coherence is an important tool to describe and compare the performance of various sensing matrices. Lower coherence implies higher reconstruction accuracy. The coherence of proposed measurement matrices is analyzed and derived to be smaller than the corresponding Gaussian and Bernoulli random matrices. Simulation experiments show that the proposed matrices outperform the corresponding Gaussian, Bernoulli, binary and chaotic bipolar matrices in reconstruction accuracy. Meanwhile, the proposed matrices can reduce the reconstruction time compared with their Gaussian counterpart. Moreover, the proposed matrices are very efficient for sensing performance, memory, complexity and hardware realization, which is beneficial to practical CS.
\end{abstract}

Keywords: Compressed sensing, measurement matrix, coherence, binary sequence family

The research is supported by National Nature Science Foundation of China (Nos. 11871109, 61802428, 11675021), NSAF Joint Fund (No. U1830107) and Science Challenge Project (TZ2018001). 


\section{Introduction}

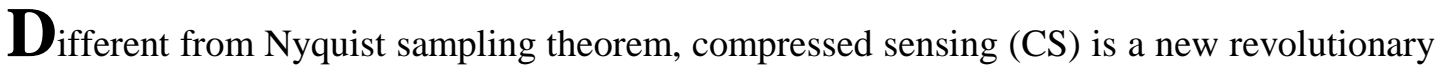
signal sampling framework proposed by Candès, Romberg, Tao and Donoho in 2006 [1, 2]. It can improve the sampling efficiency by sampling sparse signals at a rate far lower than the Nyquist rate. Its core is to exploit measurement matrix to project an original high-dimensional sparse signal onto a lower-dimensional space. By utilizing the sparsity property, the original high-dimensional sparse signal can be reconstructed accurately from the lower-dimensional measurement vector with high probability by solving an optimization problem. The new idea of CS has caused the extensive attention of academic circles and has been applied to various research areas, such as signal processing, big data, wireless network, image encryption and computed tomography.

The process of CS can be viewed as having two stages: data sampling and signal recovery. In CS theory, the design of the measurement matrix serves an important role. In data sampling, if the reconstruction accuracy remains unchanged, a better measurement matrix can result in a smaller number of measurements. For signal recovery, if the number of measurements remain unchanged, a better measurement matrix can result in a higher reconstruction accuracy. The property of measurement matrix decides whether or not all the significant information of original signal is captured and preserved by the projected measurements during the dimensionality reduction. Restricted Isometry Property (RIP) is an important criteria proposed by Candes and Tao [3]. As long as the measurement matrix satisfies RIP, the original signal can be reconstructed accurately from the lower-dimensional measurement vector with high probability. Coherence is another important criteria to construct CS matrices. Bourgain et al. [4] related the coherence and the RIP: low coherence implies the RIP. RIP [5-9] and coherence [10-22] are both important tools to analyze the property of measurement matrices. In this paper, coherence will be adopted to analyze the property of proposed measurement matrices, because it is computed more easily.

Existing measurement matrices can be classified into two categories: random measurement matrices and deterministic measurement matrices. In scientific research, the most widely used measurement matrices are random matrices, such as Gaussian or Bernoulli ones. However, in random matrices, the value of every element is independent and identically distributed (i.i.d.) from certain probability distribution, where randomness exists. In the generation process of a random matrix, all elements should be stored and the process is repeated when a new realization is needed, which would cost lots of storage resources. The generation of random number has very high hardware requirement, thus limiting the practical CS applications. These deficiencies can be overcome by deterministic measurement matrices, where all elements are precomputed and deterministic. Compared with the random matrices, the deterministic matrices can get rid of the randomness. In the generation process of a deterministic matrix, the computation of every element may require many complex mathematical operations, but all elements can be precomputed and generated on the fly only once, thus providing storage efficiency. In recent years, many researchers have utilizing some techniques to construct deterministic measurement matrices. In [10], $\mathrm{Li}$ and $\mathrm{Ge}$ constructed deterministic measurement matrices based on near orthogonal systems. In [11], Zeng et al introduced a deterministic construction named TSCM, which combines an orthonormal matrix and a chaotic-based Toeplitz one. In [12], Zhang et al constructed a lass of sparse binary deterministic measurement matrices by using the protograph low-density parity-check (LDPC) 
codes. In [13], Zhang et al presented deterministic bipolar measurement matrices arising from Legendre sequences. In [14], Tian et al proposed a deterministic construction for orthogonal-gradient measurement matrix based on the equiangular tight frame theory. In [15], Naidu et al constructed deterministic measurement matrices based on Euler Squares. In [16], Sasmal et al proposed a specialized composition rule based on the properties of existing binary matrices to produce an optimal deterministic binary CS matrices. In [17], Naidu et al related the construction of deterministic measurement matrices to the extremal set theory. In [18], Lu et al studied the optimal construction of deterministic binary CS matrix with arbitrarily given size by using the idea from bipartite graph. In [19, 20], Gan et al constructed deterministic measurement matrices based on Chebyshev chaotic sequence and topologically conjugate chaotic systems, respectively. In [21], Liu et al proposed deterministic measurement matrices based on Bose balanced incomplete block designs and used the embedding operation to develop more flexibility. In [22], Wang et al provided deterministic CS matrices based on optimal codebooks and specific codes. In [23], Liu et al involved the deterministic binary LDPC measurement matrices from complete protographs. In [24], Wang et al involved the deterministic measurement matrices by second-order Reed-Muller sequences. In [25], Hsieh et al designed a deterministic measurement matrix inspired from sparse fast Fourier transform. In [26], Fardad et al designed a low complexity hardware for generating a deterministic measurement matrix based on the Euclidian geometry LDPC code construction.

In this paper, based on the binary sequence family (BSF) in [27], we construct a class of deterministic bipolar measurement matrices named BSFDBM. The trace representative function is first chosen to produce the BSF. And then, by numeric conversion, the BSF is converted to the corresponding bipolar sequence family. The above process is repeated to generate another bipolar sequence family. By putting all sequences of the two bipolar sequence family together as column vectors, the proposed BSFDBM matrix is finally obtained.

The linear feedback shift register (LFSR) implementation of BSFDBM matrices is also given and the proposed BSFDBM matrices are proved to have smaller coherence than the corresponding Gaussian and Bernoulli random matrices. Moreover, the corresponding practical features of BSFDBM are analyzed and compared. Simulation experiments show that the proposed BSFDBM matrices outperform their Gaussian, Bernoulli, binary [18] and chaotic bipolar [9] counterparts in reconstruction accuracy with respect to one-dimensional sparse signals and different kinds of images. Meanwhile, the proposed BSFDBM matrices can reduce the reconstruction time compared with their Gaussian counterpart.

The remainder of this paper is organized as follows. Section 2 introduces the basic theory about CS and finite field. Section 3 presents deterministic construction procedure of BSFDBM matrices and related LFSR implementation. Section 4 uses the coherence to analyze the proposed BSFDBM matrices and compares the practical features of BSFDBM with other constructions. Numerical simulations are given to investigate the performance of proposed BSFDBM matrices in Section 5. Finally, Section 6 concludes this paper.

\section{Preliminaries}

\subsection{Compressed Sensing}

Suppose $\mathbf{x}=\left\{x_{i}\right\}_{i=1}^{N} \in \mathbf{R}^{N}$ is a $k$ - sparse original signal, where $\|\mathbf{x}\|_{0}=\left|\left\{i \mid x_{i} \neq 0\right\}\right| \leq k$. The observation signal $\mathbf{y} \in \mathbf{R}^{M}$ is obtained from its lower-dimensional linear projection, where 
$M \ll<$. The mathematical relationship between $\mathbf{x} \in \mathbf{R}^{N}$ and $\mathbf{y} \in \mathbf{R}^{M}$ can be expressed as $\mathbf{y}=\mathbf{A} \mathbf{x}$, where $\mathbf{A} \in \mathrm{R}^{\mathrm{M} \times \mathrm{N}}$ is called the measurement matrix. For CS, this linear projection process is also the data sampling process. However, the process of signal recovery is nonlinear. The original signal $\mathbf{x} \in \mathbf{R}^{N}$ can be reconstructed accurately by solving the following $l_{0}$ and $l_{1}$ minimization optimization problems given by (1) and (2), respectively, where $\|\mathbf{x}\|_{1}=\sum_{i=1}^{N}\left|x_{i}\right|$.

$$
\begin{aligned}
& \min _{\mathbf{x}}\|\mathbf{x}\|_{0} \text { subject to } \mathbf{y}=\mathbf{A x}, \\
& \min _{\mathbf{x}}\|\mathbf{x}\|_{1} \text { subject to } \mathbf{y}=\mathbf{A x},
\end{aligned}
$$

In problems (1) and (2), the sparsest estimate of $\mathbf{x}$ can be obtained by orthogonal matching pursuit (OMP) [28] and basis pursuit (BP) algorithm [29], respectively.

RIP serves an important role in CS $[2,15]$, because it establishes the equivalence between problems (1) and (2).

Coherence is another important criteria to construct CS matrices.

Definition 2.1 Suppose $\mathbf{a}_{1}, \mathbf{a}_{2}, \cdots, \mathbf{a}_{N}$ are the column vectors of matrix $\mathbf{A}$, then its coherence $\mu(\mathbf{A})$ is denoted as

$$
\mu(\mathbf{A})=\max _{1 \leq i \neq j \leq N} \frac{\left|\left\langle\mathbf{a}_{i}, \mathbf{a}_{j}\right\rangle\right|}{\left\|\mathbf{a}_{i}\right\|_{2} \cdot\left\|\mathbf{a}_{j}\right\|_{2}},
$$

where $\left\langle\mathbf{a}_{i}, \mathbf{a}_{j}\right\rangle=\mathbf{a}_{i}^{T} \mathbf{a}_{j}$.

As seen in [12,30], if $k<\frac{1}{2}\left[1+\frac{1}{\mu(\mathbf{A})}\right]$, any $k$-sparse signal $\mathbf{x}$ can be reconstructed accurately from its lower-dimensional linear measurement vector $\mathbf{y}=\mathbf{A x}$ via OMP or BP algorithm. Thus, for the design of measurement matrix $\mathbf{A}$, the upper bound of the sparsity $k$ of reconstructed signal can be increased by decreasing the coherence $\mu(\mathbf{A})$, which means an increase in reconstruction accuracy. To reconstruct the original signal with higher accuracy, it's required to decrease the coherence $\mu(\mathbf{A})$ as far as possible.

\subsection{Finite Field}

Definition 2.2 Let $\beta$ be the primitive field element for finite field $G F(q)$ with $q$ elements, then every elements of $G F(q)$ can be expressed as 0 or the powers of $\beta$, that is $G F(q)=\left\{0, \beta^{0}=1, \beta, \cdots, \beta^{q-2}\right\}$.

Among $\left\{0,1, \beta, \cdots, \beta^{q-2}\right\}$, the multiplicative group, denoted as $G F(q)^{*}$, consists of the $q-1$ nonzero elements. For describing convenience, $G F(q)=\left\{0, \beta^{0}=1, \beta, \cdots, \beta^{q-2}\right\}$ is simply expressed as $\{0,1, \cdots, q-1\}$.

Note that in above definition, the element structure of finite filed depends on the choice of the primitive element. For finite field $G F(q)$, if another primitive element $\gamma$ is chosen, we can obtain a new element structure for $G F(q)$.

Definition 2.3 Suppose that $m$ and $n$ are two positive integers, where $m$ is the factor of $n$. 
The trace function from $G F\left(2^{n}\right)$ to $G F\left(2^{m}\right)$, denoted as $\operatorname{Tr}_{m}^{n}(x)$, is

$$
\operatorname{Tr}_{m}^{n}(x)=x+x^{2^{m}}+\ldots+x^{2^{m\left(\frac{n}{m}-1\right)}}, x \in G F\left(2^{n}\right) .
$$

When $m=1, G F\left(2^{m}\right)=G F(2)=\{0,1\}$. For describing convenience, $\operatorname{Tr}_{1}^{n}(x)$ is simply denoted as $\operatorname{Tr}(x)$.

Definition 2.4 Let $\beta$ be a primitive element of finite field $G F\left(2^{n}\right)$ and its primitive polynomial is $g(x)$ over $G F(2)$ of degree $n$. All conjugate elements of $\beta^{i}$ with respect to $G F(2)$ are different elements of the set $\left\{\beta^{i 2^{j}}\right\}_{j=0}^{n-1}$. Let $\beta^{i 2^{d(i)}}=\beta^{i}$. Then, by using the results from finite field, $\left\{\beta^{i 2^{j}}\right\}_{j=d(i)}^{n-1} \subset\left\{\beta^{i 2^{j}}\right\}_{j=0}^{d(i)-1}$ is obtained. Therefore, $d(i)$ is the number of conjugate elements of $\beta^{i}$ and all conjugate elements of $\beta^{i}$ can be expressed as $\left\{\beta^{i 2^{j}}\right\}_{j=0}^{d(i)-1}$, that is $\left\{\beta^{i 2^{0}}=\beta^{i}, \beta^{i 2}, \cdots, \beta^{i 2^{d(i)-1}}\right\}$. The minimal polynomial of $\beta^{i}$ over $G F(2)$, denoted as $g_{i}(x)$, is

$$
g_{i}(x)=\prod_{j=0}^{d(i)-1}\left(x-\beta^{i 2^{j}}\right)
$$

\section{Construction and Implementation of BSFDBM}

\subsection{Construction of BSFDBM}

The proposed BSFDBM matrices are a class of $\left(2^{n}-1\right) \times 2^{n+1}$ deterministic bipolar matrices composed of $\{1,-1\}$ elements, where $n \geq 3$. The concrete construction procedure of BSFDBM matrices is as follows:

Step-1: According to given signal length $N=2^{n+1}$, judge $n$ being odd or even and then choose the trace representative function (6) or (7) given by [27]. If $n$ is odd, let $n=2 l+1$ and choose (6); if $n$ is even, let $n=2 l$ and choose (7), where $x \in G F\left(2^{n}\right)^{*}, \lambda \in G F\left(2^{n}\right)$.

$$
\begin{gathered}
f_{\lambda}(x)=\operatorname{Tr}(\lambda x)+\sum_{i=1}^{l} \operatorname{Tr}\left(x^{1+2^{i}}\right) \\
f_{\lambda}(x)=\operatorname{Tr}(\lambda x)+\sum_{i=1}^{l-1} \operatorname{Tr}\left(x^{1+2^{i}}\right)+\operatorname{Tr}_{1}^{l}\left(x^{1+2^{l}}\right)
\end{gathered}
$$

Step-2: Select a primitive field element $\beta$ for $G F\left(2^{n}\right)$. Let $b_{t}^{\lambda}=f_{\lambda}\left(\beta^{t}\right)$, where $t \in\left\{0,1, \cdots 2^{n}-2\right\}$, and $\lambda \in G F\left(2^{n}\right)$. The sequence $\left\{b_{t}^{\lambda}\right\}_{t=0}^{2^{n}-2}=\left\{f_{\lambda}\left(\beta^{t}\right)\right\}_{t=0}^{2^{n}-2}$, denoted as $\mathbf{b}^{\lambda}$, is a binary pseudo-random sequence of period $2^{n}-1$. The binary sequence set $\left\{\mathbf{b}^{\lambda} \mid \lambda \in G F\left(2^{n}\right)\right\}$ constitutes the BSF in [27]. By inputting every element of binary sequence $\mathbf{b}^{\lambda}=\left\{b_{t}^{\lambda}\right\}_{t=0}^{2^{n}-2}$ into the numeric conversion function (8), we can obtain the associated bipolar pseudo-random sequence $\mathbf{c}^{\lambda}=\left\{c_{t}^{\lambda}\right\}_{t=0}^{2^{n}-2}$. 


$$
c_{t}^{\lambda}=(-1)^{b_{t}^{\lambda}}=\left\{\begin{array}{l}
1, \quad b_{t}^{\lambda}=0 \\
-1, b_{t}^{\lambda}=1
\end{array}\right.
$$

For given parameter $\lambda$, the bipolar sequence $\mathbf{c}^{\lambda}=\left\{c_{t}^{\lambda}\right\}_{t=0}^{2^{n}-2}$ is deterministic. By putting together all sequences of $\left\{\mathbf{c}^{\lambda} \mid \lambda \in G F\left(2^{n}\right)\right\}$ as column vectors, we can obtain a $\left(2^{n}-1\right) \times 2^{n}$ matrix $\mathbf{A}_{1}$, which is given by

$$
\mathbf{A}_{1}=\left[\begin{array}{cccc}
c_{0}^{0} & c_{0}^{1} & \cdots & c_{0}^{2^{n}-1} \\
c_{1}^{0} & c_{1}^{1} & \cdots & c_{1}^{2^{n}-1} \\
\vdots & \vdots & \ddots & \vdots \\
c_{2^{n}-2}^{0} & c_{2^{n}-2}^{1} & \cdots & c_{2^{n}-2}^{2^{n}-1}
\end{array}\right] .
$$

Step-3: Select another primitive field element $\gamma$ for $G F\left(2^{n}\right)$. Let $d_{t}^{\lambda}=f_{\lambda}\left(\gamma^{t}\right)$, where $t \in\left\{0,1, \cdots 2^{n}-2\right\}$ and $\lambda \in G F\left(2^{n}\right)$. Repeat the process of Step-2 and we can obtain corresponding bipolar sequence family $\left\{\mathbf{h}^{\lambda}=\left\{h_{t}^{\lambda}\right\}_{t=0}^{2^{n}-2} \mid \lambda \in G F\left(2^{n}\right)\right\}$ and corresponding matrix $\mathbf{A}_{2} \in \mathbf{R}^{\left(2^{n}-1\right) \times 2^{n}}$. The matrix $\mathbf{A}_{2}$ has the following form

$$
\mathbf{A}_{2}=\left[\begin{array}{cccc}
h_{0}^{0} & h_{0}^{1} & \cdots & h_{0}^{2^{n}-1} \\
h_{1}^{0} & h_{1}^{1} & \cdots & h_{1}^{2^{n}-1} \\
\vdots & \vdots & \ddots & \vdots \\
h_{2^{n}-2}^{0} & h_{2^{n}-2}^{1} & \cdots & h_{2^{n}-2}^{2^{n}-1}
\end{array}\right]
$$

Step-4: Concatenate the above two matrices $\mathbf{A}_{1} \in \mathbf{R}^{\left(2^{n}-1\right) \times 2^{n}}$ and $\mathbf{A}_{2} \in \mathbf{R}^{\left(2^{n}-1\right) \times 2^{n}}$ in column extension form to obtain the proposed BSFDBM matrix $\mathbf{A}$ of size $\left(2^{n}-1\right) \times 2^{n+1}$. It has the following form

$$
\begin{aligned}
& \mathbf{A}=\left[\mathbf{A}_{1} \mid \mathbf{A}_{2}\right] \\
& =\left[\begin{array}{cccc|cccc}
c_{0}^{0} & c_{0}^{1} & \cdots & c_{0}^{2^{n}-1} & h_{0}^{0} & h_{0}^{1} & \cdots & h_{0}^{2^{n}-1} \\
c_{1}^{0} & c_{1}^{1} & \cdots & c_{1}^{2^{n}-1} & h_{1}^{0} & h_{1}^{1} & \cdots & h_{1}^{2^{n}-1} \\
\vdots & \vdots & \ddots & \vdots & \vdots & \vdots & \ddots & \vdots \\
c_{2^{n}-2}^{0} & c_{2^{n}-2}^{1} & \cdots & c_{2^{n}-2}^{2^{n}-1} & h_{2^{n}-2}^{0} & h_{2^{n}-2}^{1} & \cdots & h_{2^{2^{n}}-1}^{1}
\end{array}\right]
\end{aligned}
$$

From the construction, it is seen that the BSFDBM matrices have the sampling rate $\left(2^{n}-1\right) / 2^{n+1} \approx 0.5$. The BSFDBM matrix $\mathbf{A} \in \mathbf{R}^{\left(2^{n}-1\right) \times 2^{n+1}}$ is composed of two submatrices $\mathbf{A}_{1} \in \mathbf{R}^{\left(2^{n}-1\right) \times 2^{n}}$ and $\mathbf{A}_{2} \in \mathbf{R}^{\left(2^{n}-1\right) \times 2^{n}}$, each of which corresponds to a bipolar sequence family. The two bipolar sequence families have similar generation process, of which the only difference lies in the choice of the primitive field element. Without loss of generality, in the following section, we present the implementation of the bipolar sequence family corresponding to $\mathbf{A}_{1} \in \mathbf{R}^{\left(2^{n}-1\right) \times 2^{n}}$.

Remark 1: For the BSFDBM matrix, the row size is the period of the associated binary sequence, and the column size is twice the family size of associated BSF. For the BSFDBM 
matrix, some columns can be discarded to vary the sampling rate.

\subsection{LFSR Implementation of BSFDBM}

In this section, we present the implementation of sequence in the bipolar sequence family corresponding to $\mathbf{A}_{1} \in \mathbf{R}^{\left(2^{n}-1\right) \times 2^{n}}$.

For odd $n=2 l+1$, any column vector of $\mathbf{A}_{1} \in \mathbf{R}^{\left(2^{n}-1\right) \times 2^{n}}$ can be obtained by first adding $(l+1)$ m-sequences and then converting the resultant summing sequence using numeric conversion in (8). Hence, $\mathbf{A}_{1} \in \mathbf{R}^{\left(2^{n}-1\right) \times 2^{n}}$ is easy to implement by summing LFSR outputs and using numeric converter.

For odd $n=2 l+1$, $(l+1)$ n-stage LFSRs are required to implement sequences in the BSF corresponding to $\mathbf{A}_{1} \in \mathbf{R}^{\left(2^{n}-1\right) \times 2^{n}}$. The $(l+1)$ LFSRs have different characteristic polynomials for generating cyclically distinct m-sequences. Let the finite field $G F\left(2^{n}\right)$ be generated by a primitive element $\beta$ satisfying the primitive polynomial $g(x)$ over $G F(2)$ of degree $n$. For $1 \leq i \leq l$, compute $g_{i}(x)$, which is denoted as the minimal polynomial of $\beta^{1+2^{i}}$ over $G F(2)$. For LFSR 0, the characteristic polynomial is set to be $g(x)$ and the initial state can be arbitrary including 0 . For LFSR $i$ with $1 \leq i \leq l$, the characteristic polynomial is set to be $g_{i}(x)$ and the initial state is given by $\left\{\operatorname{Tr}\left(\beta^{\left(1+2^{i}\right) j}\right)\right\}_{j=0}^{n-1}$, which is fixed. According to different initial states of n-stage LFSR $0,2^{n}$ cyclically distinct binary sequences are generated to constitute the required BSF. The related LFSR implementation of $\mathbf{A}_{1}$ is simply shown in Fig. 1.

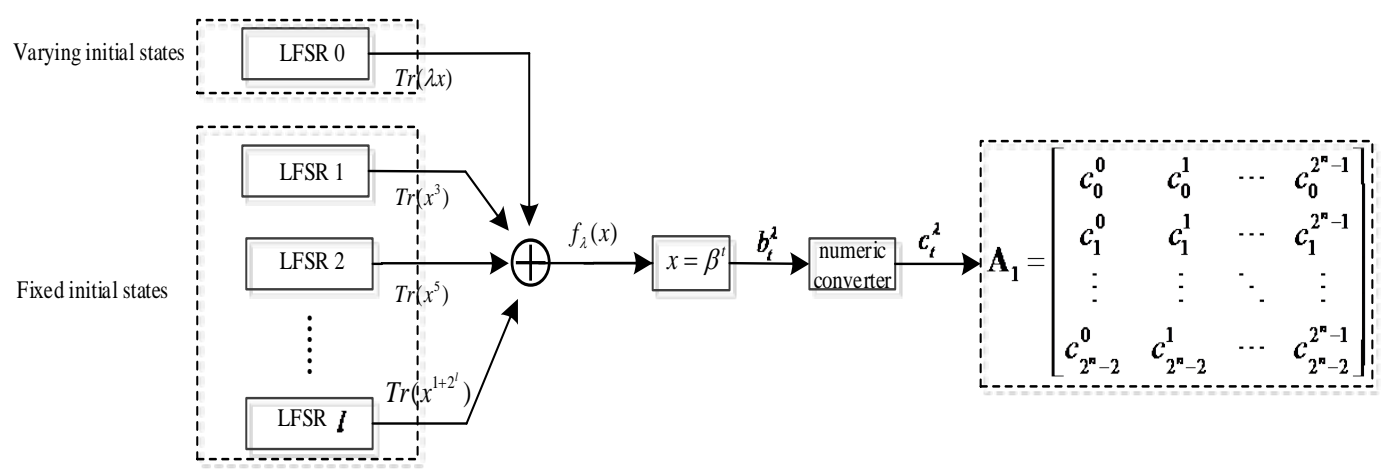

Fig. 1. LFSR implementation of corresponding matrix

For even $n=2 l$, the BSF corresponding to $\mathbf{A}_{1} \in \mathbf{R}^{\left(2^{n}-1\right) \times 2^{n}}$ can be implemented similarly to that case of odd $n$, except that the size of LFSR $l$ is $n / 2$.

From above implementation, it can be seen that the proposed BSFDBM matrices are very efficient for hardware realization, which is extremely easy via LFSR structures. 


\section{Performance Analysis}

\subsection{Coherence Analysis}

Coherence is an important criteria to describe the property of matrices. For CS matrices, decreasing the coherence leads to an increase in reconstruction accuracy. This section first gives the coherence of proposed BSFDBM matrices and then derives that the coherence of BSFDBM matrices is smaller than the corresponding Gaussian and Bernoulli random matrices.

In order to analyze the coherence of the BSFDBM matrices, we first introduce the following two definitions and one lemma [27].

Definition 4.1 For two different binary sequences $\mathbf{a}=\left(a_{0}, a_{1}, \cdots, a_{v}\right)$ and $\mathbf{b}=\left(b_{0}, b_{1}, \cdots, b_{v}\right)$ of period $v$, the cross-correlation of $\mathbf{a}$ and $\mathbf{b}$ is defined as $C_{\mathbf{a}, \mathbf{b}}(\tau)=\sum_{i=0}^{v-1}(-1)^{a_{i}+b_{i+\tau}}$ for $0 \leq \tau \leq v-1$, where $i+\tau$ is computed modulo $v$. If $\mathbf{a}$ and $\mathbf{b}$ are cyclically equivalent, $C_{\mathbf{a}, \mathbf{b}}(\tau)$ is the auto-correlation of $\mathbf{a}$ or $\mathbf{b}$.

Definition 4.2 Let $S=\left\{\mathbf{s}^{(0)}, \mathbf{s}^{(1)}, \cdots, \mathbf{s}^{(r-1)}\right\}$ be the set of $r$ cyclically distinct binary sequences of period $v$. Define $C_{\max }=\max \left|C_{\mathbf{s}^{(i)}, \mathbf{s}^{(j)}}(\tau)\right|$ for $0 \leq \tau \leq v-1$ and $0 \leq i, j \leq r-1$, where $\tau \neq 0$ if $i=j$. Obviously, $C_{\max }$ is the maximum value among all auto- and cross-correlations of the sequences in $S . C_{\max }$ is also called the maximum correlation magnitude of $S$.

Lemma 4.1 For odd $n$, the cross-correlation of any two binary sequences $\mathbf{a}$ and $\mathbf{b}$ in BSF given by (6) is $C_{\mathbf{a}, \mathbf{b}}(\tau) \in\left\{-1,-1 \pm 2^{(n+1) / 2}\right\}$ and the maximum correlation $C_{\max }$ is $1+2^{(\mathrm{n}+1) / 2}$.

For even $n$, the cross-correlation of any two binary sequences $\mathbf{a}$ and $\mathbf{b}$ in BSF given by (7) is $C_{\mathbf{a}, \mathbf{b}}(\tau) \in\left\{-1,-1 \pm 2^{n / 2},-1 \pm 2^{n / 2+1}\right\}$ and the maximum correlation $C_{\max }$ is $1+2^{n / 2+1}$.

Theorem 4.1 Let $\mathbf{A}$ be a $\left(2^{n}-1\right) \times 2^{n+1}(n \geq 3)$ BSFDBM matrix. If $n$ is odd, $\mu(\mathbf{A})=\frac{1+2^{(n+1) / 2}}{2^{n}-1}$; if $n$ is even, $\mu(\mathbf{A})=\frac{1+2^{n / 2+1}}{2^{n}-1}$.

Proof: For matrix $\mathbf{A} \in \mathbf{R}^{\left(2^{n}-1\right) \times 2^{n+1}}$, we have

$$
\mu(\mathbf{A})=\max _{1 \leq i \neq j \leq 2^{n+1}} \frac{\left\langle\mathbf{A}^{i}, \mathbf{A}^{j}\right\rangle \mid}{\left\|\mathbf{A}^{i}\right\|_{2} \cdot\left\|\mathbf{A}^{j}\right\|_{2}}
$$

where $\mathbf{A}^{i}$ is the $i$ th column of $\mathbf{A}$. Note that sequences $\mathbf{A}^{i}$ and $\mathbf{A}^{j}$ are bipolar sequence of length $2^{n}-1$.Thus

$$
\left\|\mathbf{A}^{i}\right\|_{2}=\left\|\mathbf{A}^{j}\right\|_{2}=\left(2^{n}-1\right)^{1 / 2}
$$

As seen in Section 3, the BSFDBM matrix $\mathbf{A}=\left[\mathbf{A}_{1} \mid \mathbf{A}_{2}\right]$ is composed of two submatrices $\mathbf{A}_{1} \in \mathbf{R}^{\left(2^{n}-1\right) \times 2^{n}}$ and $\mathbf{A}_{2} \in \mathbf{R}^{\left(2^{n}-1\right) \times 2^{n}}$, each of which corresponds to a primitive field element. 
For $s=1,2, \mathbf{A}_{s} \in \mathbf{R}^{\left(2^{n}-1\right) \times 2^{n}}$ has a $\operatorname{BSF}\left\{\mathbf{b}^{\lambda}(s) \mid \lambda \in G F\left(2^{n}\right)\right\}$ and a associated bipolar sequence family $\left\{\mathbf{c}^{\lambda}(s) \mid \lambda \in G F\left(2^{n}\right)\right\}$. The $i$ th column of $\mathbf{A}_{s}$, denoted as $\mathbf{A}_{s}^{i}$, is the bipolar sequence $\mathbf{c}^{i}(s)$ in $\left\{\mathbf{c}^{\lambda}(s) \mid \lambda \in G F\left(2^{n}\right)\right\}$.

Depending on $i$ and $j$, the calculation of $\max _{1 \leq i \neq j \leq 2^{n+1}}\left|\left\langle\mathbf{A}^{i}, \mathbf{A}^{j}\right\rangle\right|$ can be classified into two cases.

Case 1: $1 \leq i, j \leq 2^{n}$ or $2^{\mathrm{n}}+1 \leq i, j \leq 2^{n+1}$.

In this case, $\max _{1 \leq i \neq j \leq 2^{n+1}}\left|\left\langle\mathbf{A}^{i}, \mathbf{A}^{j}\right\rangle\right|=\max _{1 \leq i \neq j \leq 2^{n}}\left|\left\langle\mathbf{A}_{1}^{i}, \mathbf{A}_{1}^{j}\right\rangle\right|=\max _{1 \leq i \neq j \leq 2^{n}}\left|\left\langle\mathbf{A}_{2}^{i}, \mathbf{A}_{2}^{j}\right\rangle\right|$.

Assume that $\mathbf{b}^{i}(s)=\left\{b_{t}^{i}(s)\right\}_{t=0}^{2^{n}-2}$ and $\mathbf{b}^{j}(s)=\left\{b_{t}^{j}(s)\right\}_{t=0}^{2^{n}-2}$ are any two binary sequences in $\left\{\mathbf{b}^{\lambda}(s) \mid \lambda \in G F\left(2^{n}\right)\right\}$. From (8), the associated two bipolar sequences $\mathbf{c}^{i}(s)=\left\{c_{t}^{i}(s)\right\}_{t=0}^{2^{n}-2}$ and $\mathbf{c}^{j}(s)=\left\{c_{t}^{j}(s)\right\}_{t=0}^{2^{n}-2}$ are obtained.

We have $\left\langle\mathbf{A}_{s}^{i}, \mathbf{A}_{s}^{j}\right\rangle=\left\langle\mathbf{c}^{i}(s), \mathbf{c}^{j}(s)\right\rangle=\sum_{t=0}^{2^{n}-2} c_{t}^{i}(s) c_{t}^{j}(s)=\sum_{t=0}^{2^{n}-2}(-1)^{b_{t}^{i}(s)+b_{t}^{j}(s)}=C_{\mathbf{b}^{i}(s), \mathbf{b}^{j}(s)}(0)$ for $s=1,2$.

Based on Lemma 4.1, we derive that if $n$ is odd,

$$
\begin{aligned}
\max _{1 \leq i \neq j \leq 2^{n}} \mid\left\langle\mathbf{A}_{1}^{i}, \mathbf{A}_{1}^{j}\right\rangle & =\max _{1 \leq i \neq j \leq 2^{n}}\left|\left\langle\mathbf{A}_{2}^{i}, \mathbf{A}_{2}^{j}\right\rangle\right| \\
& =\max _{1 \leq i \neq j \leq 2^{n}}\left|C_{\mathbf{b}^{i}(2), \mathbf{b}^{j}(2)}(0)\right| \\
& =\max \left|-1,-1 \pm 2^{(n+1) / 2}\right| \\
& =1+2^{(n+1) / 2}
\end{aligned}
$$

In a similar derivation process for odd $n$, for even $n$, we have

$$
\max _{1 \leq i \neq j \leq 2^{n}}\left|\left\langle\mathbf{A}_{1}^{i}, \mathbf{A}_{1}^{j}\right\rangle\right|=\max _{1 \leq i \neq j \leq 2^{n}}\left|\left\langle\mathbf{A}_{2}^{i}, \mathbf{A}_{2}^{j}\right\rangle\right|=1+2^{n / 2+1}
$$

Case 2: $1 \leq i \leq 2^{n}$ and $2^{\mathrm{n}}+1 \leq j \leq 2^{n+1}$.

In this case, $\max _{1 \leq i \neq j \leq 2^{n+1}}\left|\left\langle\mathbf{A}^{i}, \mathbf{A}^{j}\right\rangle\right|=\max _{1 \leq i, j \leq 2^{n}} \mid\left\langle\mathbf{A}_{1}^{i}, \mathbf{A}_{2}^{j}\right\rangle$.

Assume that $\mathbf{b}^{i}(1)=\left\{b_{t}^{i}(1)\right\}_{t=0}^{2^{n}-2}$ and $\mathbf{b}^{j}(2)=\left\{b_{t}^{j}(2)\right\}_{t=0}^{2^{n}-2}$ are any binary sequences of $\left\{\mathbf{b}^{\lambda}(1) \mid \lambda \in G F\left(2^{n}\right)\right\}$ and $\left\{\mathbf{b}^{\lambda}(2) \mid \lambda \in G F\left(2^{n}\right)\right\}$, respectively. From (8), the associated two bipolar sequences $\mathbf{c}^{i}(1)=\left\{c_{t}^{i}(1)\right\}_{t=0}^{2^{n}-2}$ and $\mathbf{c}^{j}(2)=\left\{c_{t}^{j}(2)\right\}_{t=0}^{2^{n}-2}$ are obtained. We have $\left\langle\mathbf{A}_{1}^{i}, \mathbf{A}_{2}^{j}\right\rangle=\left\langle\mathbf{c}^{i}(1), \mathbf{c}^{j}(2)\right\rangle=\sum_{t=0}^{2^{n}-2} c_{t}^{i}(1) c_{t}^{j}(2)=\sum_{t=0}^{2^{n}-2}(-1)^{b_{t}^{i}(1)+b_{t}^{j}(2)}=C_{\mathbf{b}^{i}(1), \mathbf{b}^{j}(2)}(0)$.

The sequence families $\left\{\mathbf{c}^{\lambda}(1) \mid \lambda \in G F\left(2^{n}\right)\right\}$ and $\left\{\mathbf{c}^{\lambda}(2) \mid \lambda \in G F\left(2^{n}\right)\right\}$ have similar generation process, of which the only difference lies in the choice of the primitive field element. By using the results from finite field, the two bipolar sequence families $\left\{\mathbf{c}^{\lambda}(1) \mid \lambda \in G F\left(2^{n}\right)\right\}$ and $\left\{\mathbf{c}^{\lambda}(2) \mid \lambda \in G F\left(2^{n}\right)\right\}$ are cyclically equivalent. 
Correspondingly, $\quad\left\{\mathbf{b}^{\lambda}(1) \mid \lambda \in G F\left(2^{n}\right)\right\} \quad$ and $\quad\left\{\mathbf{b}^{\lambda}(2) \mid \lambda \in G F\left(2^{n}\right)\right\} \quad$ are cyclically equivalent. Therefore, for $\mathbf{b}^{j}(2) \in\left\{\mathbf{b}^{\lambda}(2) \mid \lambda \in G F\left(2^{n}\right)\right\}$, there must exist a corresponding cyclically equivalent sequence $\mathbf{b}^{l}(1)$ in $\left\{\mathbf{b}^{\lambda}(1) \mid \lambda \in G F\left(2^{n}\right)\right\}$. Thus, there exists a integer $\tau$ to make $C_{\mathbf{b}^{i}(1), \mathbf{b}^{j}(2)}(0)=C_{\mathbf{b}^{i}(1) \mathbf{b}^{\prime}(1)}(\tau)$ hold. According to Definition 4.1, $\tau$ means the phase shift of sequence. Based on Lemma 4.1, we derive that if $n$ is odd,

$$
\max _{1 \leq i, j \leq 2^{n}}\left|\left\langle\mathbf{A}_{1}^{i}, \mathbf{A}_{2}^{j}\right\rangle\right|=\max _{1 \leq i, j \leq 2^{n}}\left|C_{\mathbf{b}^{i}(1), \mathbf{b}^{j}(2)}(0)\right|=\max _{1 \leq i, j \leq 2^{n}}\left|C_{\mathbf{b}^{i}(1), \mathbf{b}^{l}(1)}(\tau)\right|=1+2^{(n+1) / 2} .
$$

In a similar derivation process for odd $n$, for even $n$, we have $\max _{1 \leq i, j \leq 2^{n}}\left|\left\langle\mathbf{A}_{1}^{i}, \mathbf{A}_{2}^{j}\right\rangle\right|=1+2^{n / 2+1}$.

Combing the Cases 1 and 2, we have the conclusion that if $n$ is odd,

$$
\begin{aligned}
& \max _{1 \leq i \neq j \leq 2^{n+1}}\left|\left\langle\mathbf{A}^{i}, \mathbf{A}^{j}\right\rangle\right| \\
& =\max \left\{\max _{1 \leq i \neq j \leq 2^{n}}\left|\left\langle\mathbf{A}_{1}^{i}, \mathbf{A}_{1}^{j}\right\rangle\right|, \max _{1 \leq i, j \leq 2^{n}} \mid\left\langle\mathbf{A}_{1}^{i}, \mathbf{A}_{2}^{j}\right\rangle\right\} \\
& =\max \left\{1+2^{(n+1) / 2}, 1+2^{(n+1) / 2}\right\} \\
& =1+2^{(n+1) / 2} .
\end{aligned}
$$

Similarly, if $n$ is even, $\max _{1 \leq i \neq j \leq 2^{n+1}}\left|\left\langle\mathbf{A}^{i}, \mathbf{A}^{j}\right\rangle\right|=1+2^{n / 2+1}$.

Theorem 4.1 can be proved after substituting above conclusions and (13) into (12).

To compare the coherence of the BSFDBM matrices with the Gaussian and Bernoulli matrices, we need the following two lemmas [31].

Lemma 4.2 Let $\left\{x_{i}\right\}_{i=1}^{p}$ and $\left\{y_{i}\right\}_{i=1}^{p}$ be sequences of i.i.d. zero-mean Gaussian random variables with variance $\sigma^{2}$. Then $\operatorname{Pr}\left(\left|\sum_{i=1}^{p} x_{i} y_{i}\right| \geq t\right) \leq 2 \exp \left(-\frac{t^{2}}{4 \sigma^{2}\left(p \sigma^{2}+t / 2\right)}\right)$.

Lemma 4.3 Let $\left\{x_{i}\right\}_{i=1}^{p}$ and $\left\{y_{i}\right\}_{i=1}^{p}$ be sequences of i.i.d. zero-mean bounded random variables which satisfy $\left|x_{i}\right| \leq a$ and $\left|x_{i} y_{i}\right| \leq a^{2}$. Then $\operatorname{Pr}\left(\left|\sum_{i=1}^{p} x_{i} y_{i}\right| \geq t\right) \leq 2 \exp \left(-\frac{t^{2}}{2 p a^{4}}\right)$.

Theorem 4.2 For a $\left(2^{n}-1\right) \times 2^{n+1}(n \geq 3)$ BSFDBM matrix $\mathbf{A}$ and its Gaussian counterpart $\mathbf{B}, \mu(\mathbf{A})<\mu(\mathbf{B})$ holds.

Proof: Let $\mathbf{b}_{i}$ be the column vector of the matrix $\mathbf{B} \in \mathbf{R}^{\left(2^{n}-1\right) \times 2^{n+1}}$ for $1 \leq i \leq 2^{n+1}$.

Without loss of generality, we prove the theorem in case of even $n$.

Suppose that $\left\{x_{i}\right\}_{i=1}^{2^{n}-1}$ and $\left\{y_{i}\right\}_{i=1}^{2^{n}-1}$ are any two column vectors of matrix $\mathbf{B}$. Based on Lemma 4.2 with $p=2^{n}-1, t>\frac{1+2^{n / 2+1}}{2^{n}-1}$, and $\sigma^{2}=\frac{1}{2^{n}-1}$, we have

$$
\operatorname{Pr}\left(\left|\sum_{i=1}^{n} x_{i} y_{i}\right| \geq t\right) \leq 2 \exp \left\{-\frac{\left(2^{n}-1\right) t^{2}}{4+2 t}\right\} \text {. }
$$


Let $z(n, t)=2 \exp \left\{-\frac{\left(2^{n}-1\right) t^{2}}{4+2 t}\right\}$. It is easy to derive that $z(n, t)$ increases with $n$ decreasing. Thus, we have $z(n, t) \leq z(4, t)$. We can further derive that

$$
\operatorname{Pr}\left(\left|\sum_{i=1}^{2^{n}-1} x_{i} y_{i}\right| \geq t\right) \leq z(4, t)=2 \exp \left\{-\frac{15 t^{2}}{4+2 t}\right\} .
$$

We observe that $z(4, t)$ increases with $t$ decreasing. Thus, we have $z(4, t)<z\left(4, \frac{1+2^{n / 2+1}}{2^{n}-1}\right)$. Let $z_{1}(n)=z\left(4, \frac{1+2^{n / 2+1}}{2^{n}-1}\right)$. Obviously, $z_{1}(n)$ increases with $n$ decreasing.

Thus, we have $z(4, t)<z_{1}(n) \leq z_{1}(4) \approx 2 \exp (-1.0385)$. Further, we have

$$
\operatorname{Pr}\left(\left|\sum_{i=1}^{2^{n}-1} x_{i} y_{i}\right| \geq t\right)<z_{1}(4) \approx 2 \exp (-1.0385) \approx 0.708 \text {. }
$$

According to Definition 2.1, $\left|\sum_{i=1}^{2^{n}-1} x_{i} y_{i}\right|$ can characterize the coherence $\mu(\mathbf{B})$ of matrix $\mathbf{B}$ Let $S=\left\{\mathbf{b}_{1}, \mathbf{b}_{2}, \ldots, \mathbf{b}_{2^{n+1}}\right\}$. We have

$$
\mu(\mathbf{B})=\max _{\left\{x_{i}\right\},\left\{y_{i}\right\}}\left\{\sum_{i=1}^{2^{n}-1} x_{i} y_{i} \mid\left\{x_{i}\right\} \subset S,\left\{y_{i}\right\} \subset S \backslash\left\{x_{i}\right\}\right\} .
$$

Further, we have

$$
\operatorname{Pr}\left(\min _{\left\{x_{i}\right\},\left\{y_{i}\right\}}\left|2^{2^{n}-1} x_{i=1} y_{i}\right| \geq t\right)<0.708^{\frac{|s|(|S|-1)}{2}}=0.708^{2^{n}\left(2^{n+1}-1\right)} .
$$

Let $\delta_{b}(n)=0.708^{2^{n}\left(2^{n+1}-1\right)}$ with $n \geq 4$. Obviously, we can further derive that

$$
\operatorname{Pr}\left(\min _{\left\{x_{i}\right\},\left\{y_{i}\right\}}\left|\sum_{i=1}^{2^{n}-1} x_{i} y_{i}\right| \leq t\right) \geq 1-\delta_{b}(n) \approx 1 .
$$

Hence, $\mu(\mathbf{B})=\max _{\left\{x_{i}\right\},\left\{y_{i}\right\}} \sum_{i=1}^{2^{n}-1} x_{i} y_{i} \geq t$. By $t>\frac{1+2^{n / 2+1}}{2^{n}-1}$, and $\mu(\mathbf{A})=\frac{1+2^{n / 2+1}}{2^{n}-1}$, we have $\mu(\mathbf{B})>\mu(\mathbf{A})$.

Similarly, we can obtain the same conclusion in case of odd $n$. Thus, we complete the proof of Theorem 4.2.

In a similar derivation process of Theorem 4.2, we can obtain the following corollary based on the Lemma 4.3.

Corollary 4.1 For a $\left(2^{n}-1\right) \times 2^{n+1}(n \geq 3)$ BSFDBM matrix A and its Bernoulli counterpart $\mathbf{D}$ of elements $\{1,-1\}, \mu(\mathbf{A})<\mu(\mathbf{D})$ holds.

Remark 2: Theorem 4.2 and Corollary 4.1 can demonstrate that the BSFDBM matrices outperform their Gaussian and Bernoulli counterparts in reconstruction accuracy.

Based on the above work, a novel framework has been presented for constructing bipolar 
measurement matrices via BSF. Furthermore, through the coherence analysis, we have shown that the BSFDBM matrices are proper candidates for CS matrices, just as Gaussian and Bernoulli do. In fact, by this framework, more bipolar matrices can be explored by many other binary sequence families in Table 1.

Table 1. Comparison of different families of binary sequences

\begin{tabular}{|c|c|c|c|c|}
\hline Family of Sequences & Period & Family Size & $\begin{array}{c}\text { Maximum Correlation } \\
\text { Magnitude } C_{\max }\end{array}$ & $\begin{array}{c}\text { Parameter } \\
n\end{array}$ \\
\hline Gold [32] & $2^{n}-1$ & $2^{n}+1$ & $1+2^{(n+1) / 2}$ & odd \\
\hline Udaya [33] & $2^{n}-1$ & $2^{n}+1$ & $1+2^{n / 2+1}$ & even \\
\hline Rothaus [34] & $2^{n}-1$ & $2^{2 n}+2^{n}+1$ & $1+2^{(n+3) / 2}$ & odd \\
\hline $\begin{array}{c}\text { Delsarte-Goethals } \\
\text { [35] }\end{array}$ & $2\left(2^{n}-1\right)$ & $2^{2 n}-1$ & $2+2^{(n+3) / 2}$ & odd \\
\hline
\end{tabular}

Remark 3: We replace the trace representative functions (6) and (7) by functions (24) and (25) given in [27], respectively, where $x \in G F\left(2^{n}\right)^{*}, \lambda_{0}, \lambda_{1} \in G F\left(2^{n}\right)$.

$$
\begin{gathered}
f_{\lambda_{0}, \lambda_{1}}(x)=\operatorname{Tr}\left(\lambda_{0} x\right)+\operatorname{Tr}\left(\lambda_{1} x^{3}\right)+\sum_{i=2}^{l} \operatorname{Tr}\left(x^{1+2^{i}}\right) \\
f_{\lambda_{0}, \lambda_{1}}(x)=\operatorname{Tr}\left(\lambda_{0} x\right)+\operatorname{Tr}\left(\lambda_{1} x^{3}\right)+\sum_{i=2}^{l-1} \operatorname{Tr}\left(x^{1+2^{i}}\right)+\operatorname{Tr}_{1}^{l}\left(x^{1+2^{l}}\right)
\end{gathered}
$$

The BSFDBM A will become a new bipolar measurement matrix of size $\left(2^{n}-1\right) \times 2^{2 n+1}$, where $n \geq 5$. If $n$ is odd, it has the coherence $\mu(\mathbf{A})=\frac{1+2^{(n+3) / 2}}{2^{n}-1}$; if $n$ is even, $\mu(\mathbf{A})=\frac{1+2^{n / 2+2}}{2^{n}-1}$. Without complete detail, it is pointed out that this kind of bipolar matrices can also serve as CS matrices.

Remark 4: For the binary sequence families in Table 1, we can apply our method to these binary systems, and obtain a large family of bipolar CS matrices. The minimum sampling rate equals twice the family size divided by the period of the binary sequence.

\subsection{Benefit of BSFDBM}

Based on the above coherence analysis, the low coherence of BSFDBM can ensure its good sensing performance. However, for practical CS matrix, we should also consider other practical features, including memory cost, computational complexity and hardware realization. Here, we analyze and compare the practical features of BSFDBM with its counterparts (Gaussian, Bernoulli random matrices, deterministic binary matrices [18] and CsPM [9] ) in Table 2. Among them, binary matrix [18] is obtained by using the idea from bipartite graph with column degree $d=\operatorname{ceil}(\sqrt{M})$. CsPM is the Chebyshev chaotic bipolar matrix [9]. Note that operator 'ceil' rounds the elements to the nearest integers towards infinity. For a fair comparison, let $M \times N$ be the matrix size and $B$ be the number of required bits to store every decimal element. 
Table 2. The comparison of practical feature of BSFDBM

\begin{tabular}{|c|c|c|c|c|c|}
\hline Features & BSFDBM & Gaussian & Bernoulli & Binary [18] & CsPM [9] \\
\hline $\begin{array}{c}\text { Randomness or } \\
\text { deterministic }\end{array}$ & Deterministic & Randomness & Randomness & Deterministic & Deterministic \\
\hline $\begin{array}{c}\text { Memory cost } \\
\text { (bits) }\end{array}$ & $M N$ & $B M N$ & $M N$ & $M N$ & $M N$ \\
\hline Multiplier-less & Yes & No & Yes & Yes & Yes \\
\hline $\begin{array}{c}\text { Hardware- } \\
\text { friendly }\end{array}$ & Yes & No & No & Yes & Yes \\
\hline
\end{tabular}

Table 2 shows that the BSFDBM has the following practical advantages:

(1) Low memory cost: The BSFDBM consists of elements of +1 and -1 . Therefore BSFDBM requires $M N$ bits to store all elements. Compared with its random Gaussian counterparts, BSFDBM reduces the memory requirement, thus providing storage efficiency. This feature would make BSFDBM be beneficial to practical resource-limited CS applications, such as wireless body network;

(2) Low computational complexity: The proposed BSFDBM matrix being bipolar supports multiplier-less operation, fast data acquisition and recovery. For data acquisition and recovery, the arithmetic operations of BSFDBM are addition and subtraction, whereas the random Gaussian construction demands addition, subtraction and multiplication.

(3) Hardware-friendly realization: As seen in section 3.2, the implementation of BSFDBM is extremely easy by means of LFSR structures, thus providing hardware-friendly realization. However, in the random constructions (Gaussian and Bernoulli), random number generation has very high hardware requirement, which is not hardware-friendly.

From the above analysis, it can be clearly seen that, compared to its random constructions (Gaussian and Bernoulli), the proposed BSFDBM has a good tradeoff among sensing performance, memory cost, computational complexity and hardware realization.

Remark 5: From Table 2, we can see that the proposed BSFDBM has comparable practical features (memory cost, computational complexity and hardware realization) to that of the binary matrices [18] and CsPM [9]. In the following section, we will compare the sensing performance of proposed BSFDM matrices with that of binary matrices [18] and CsPM [9] via numerical simulations.

\section{Simulation and Results}

In this section, the performance of proposed BSFDBM matrices is investigated through numerical simulations with sparse signals and images. Here, the compared matrices are Gaussian random, Bernoulli random, deterministic binary matrices [18] and CsPM [9]. For Gaussian matrix, the value of every element is i.i.d. from standard normal distribution $N(0,1)$. For Bernoulli matrix, the value of every element is -1 or 1 with equal probability. As for signal recovery, the OMP algorithm is performed to profit from the lower coherence of CS matrices. 


\subsection{BSFDBM for Sparse Signals}

The reconstruction accuracy of BSFDBM matrices is compared with that of corresponding Gaussian, Bernoulli, binary and CsPM matrices in noiseless and noisy scenarios. Two types of BSFDBM matrices of size $\left(2^{n}-1\right) \times 2^{n+1}$ are generated: (i) BSFDBM matrices of size $255 \times 512$ for even $n$ and $n=8$; (ii) BSFDBM matrices of size $127 \times 256$ for odd $n$ and $n=7$.

In the simulation, the $k$-sparse $2^{n+1} \times 1$ original signals $\mathbf{x}$ with $k$ nonzero locations uniformly randomly and corresponding $k$ nonzero values taken by $N(0,1)$ are considered. For each sparsity level $k, 1000$ trials are averaged to obtain the corresponding numerical result. Let $\mathbf{x}_{R}$ be the reconstructed solution from OMP. In noiseless scenario, if $\left\|\mathbf{x}-\mathbf{x}_{R}\right\|_{2}<10^{-6}$, this reconstruction trial is declared to be successful. The percentage of successful reconstruction times is calculated as the successful reconstruction probability. In noisy scenario, additive Gaussian noise $\mathbf{e}$ is added to the signal $\mathbf{x}$, where the signal-to-noise ratio (SNR) is 30dB. The reconstruction SNR is denoted as $\operatorname{SNR}(\mathbf{x})=20 \cdot \log _{10}\left(\|\mathbf{x}\|_{2} /\left\|\mathbf{x}-\mathbf{x}_{R}\right\|_{2}\right) d B$.

Noiseless scenario: For matrices of size $255 \times 512$, Fig. 2(a) shows the probability of successful reconstruction of $k$-sparse $512 \times 1$ signals, where $40 \leq k \leq 138$. For matrices of size $127 \times 256$, Fig. 2(b) shows the probability of successful reconstruction of $k$-sparse $256 \times 1$ signals, where $15 \leq k \leq 85$.

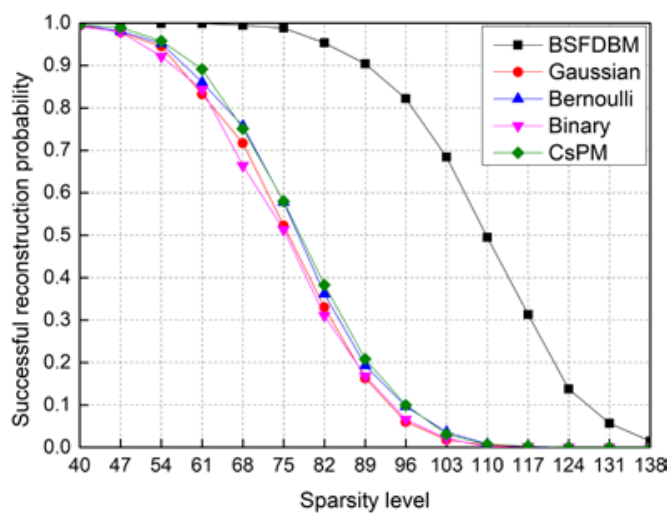

(a)

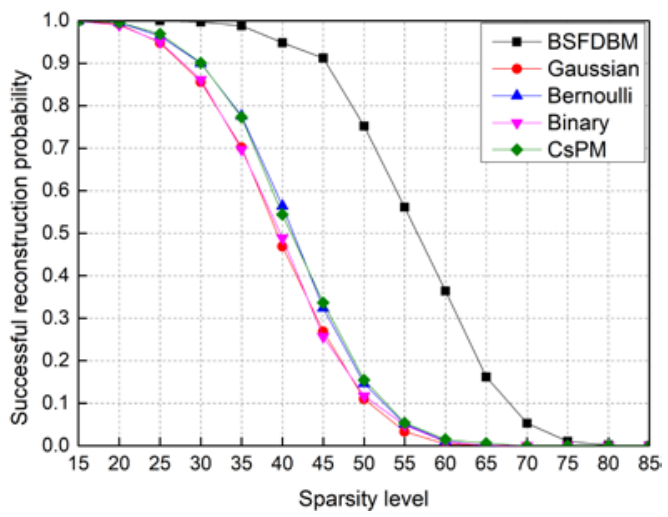

(b)

Fig. 2. Comparison of the successful reconstruction probability of noiseless sparse signals.

(a) Matrices of size $255 \times 512$, (b) Matrices of size $127 \times 256$

It can be seen from Fig. 2 that the reconstruction accuracy of BSFDBM matrix is superior to the Gaussian, Bernoulli, binary and CsPM matrices.

Noisy scenario: For matrices of size $255 \times 512$, Fig. 3(a) shows the reconstruction SNR of $k$-sparse $512 \times 1$ signals, where $40 \leq k \leq 138$. For matrices of size $127 \times 256$, Fig. 3(b) shows the reconstruction SNR of $k$-sparse $256 \times 1$ signals, where $15 \leq k \leq 85$. 


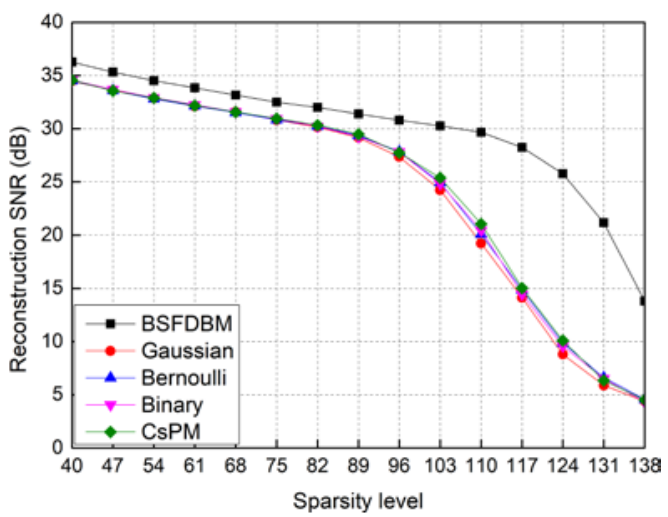

(a)

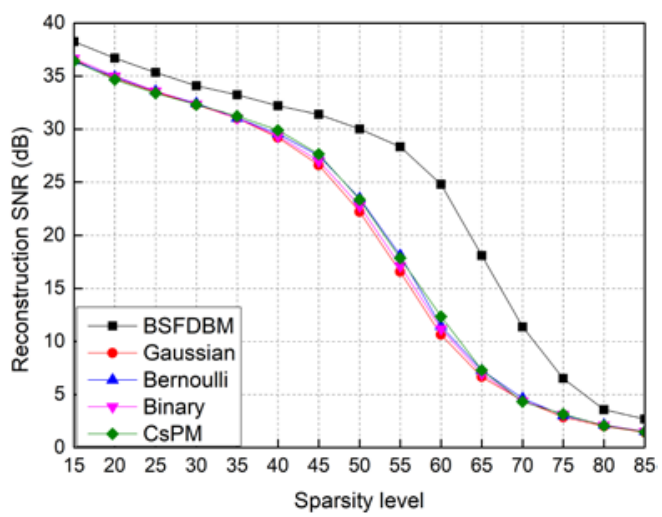

(b)

Fig. 3. Comparison of the reconstruction SNR of noisy sparse signals.

(a) Matrices of size $255 \times 512$, (b) Matrices of size $127 \times 256$

It can be seen from Fig. 3 that for all sparsity level, the BSFDBM matrix has more reconstruction SNR than its Gaussian, Bernoulli, binary and CsPM counterparts. This illustrates that the BSFDBM matrix is more robust to noise than other constructions considered.

From above two scenarios, it is observed that, for noiseless and noisy scenarios, the proposed BSFDBM matrices provide better reconstruction accuracy than the corresponding Gaussian, Bernoulli, binary and CsPM matrices.

\subsection{BSFDBM for Image Signals}

In this part, we compare the performance of image reconstruction of BSFDBM matrices with that of corresponding Gaussian, Bernoulli, binary and CsPM matrices via the block CS algorithm. As shown in Fig. 4, the test images consist of five grayscale images and five color images. The five grayscale test images are "boat" of size $256 \times 256$, "fruits" of size $256 \times 256$, "liftingbody" of size $512 \times 512$, "phantom" of size $256 \times 256$ and "cameraman" of size $256 \times 256$, whereas the five color test images are "concordaerial" of size $2036 \times 3060 \times 3$, "bone1" of size $837 \times 1242 \times 3$, "bone2" of size $692 \times 631 \times 3$, "lighthouse" of size $640 \times 480 \times 3$ and "Saturn" of size $1500 \times 1200 \times 3$. The sparsifying basis for these six images is the Daubechies 9/7 discrete wavelet transform (DWT). Considering the feature of Daubechies 9/7 DWT and the tradeoff between reconstruction time and accuracy, the block sizes $32 \times 16$ and $32 \times 32$ are selected, each of which corresponds to one type of BSFDBM matrices. To characterize the image reconstruction performance, the peak signal-to-noise ratio (PSNR) is used as the evaluation criterion. For a two-dimensional image signal $\mathbf{x}$ of size $m \times n$ with $\mathbf{x}_{R}$ be the reconstructed signal, the PSNR is denoted as $\operatorname{PSNR}(\mathbf{x})=10 \cdot \log _{10}\left(255^{2} /\left(\left\|\mathbf{x}-\mathbf{x}_{R}\right\|_{2}^{2} / m / n\right)\right) d B$.

Note that for a three-dimensional color image $\mathbf{x}$, we first convert the signal $\mathbf{x}$ to a two-dimensional grayscale signal $\mathbf{x}^{F}$ by concatenating the R, G, B components in order and column extension form. The resulting signal $\mathbf{x}^{F}$ and corresponding reconstructed signal $\mathbf{x}_{R}^{F}$ are applied to calculate $\operatorname{PSNR}\left(\mathbf{x}^{F}\right)$. 


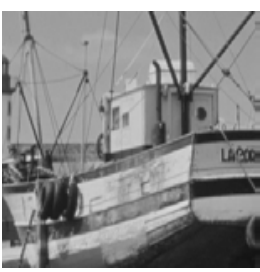

(a)

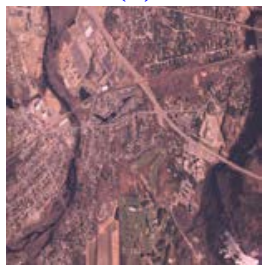

(f)

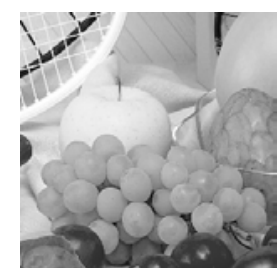

(b)

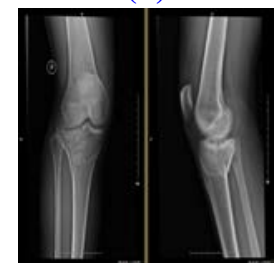

(g)

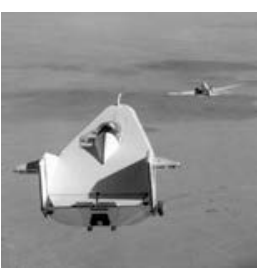

(c)

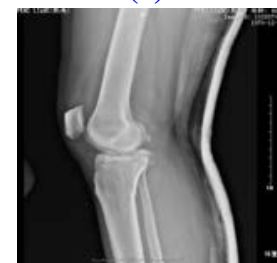

(h)

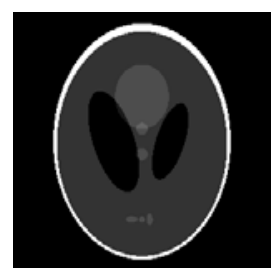

(d)

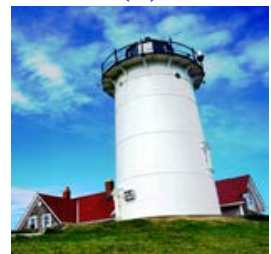

(i)

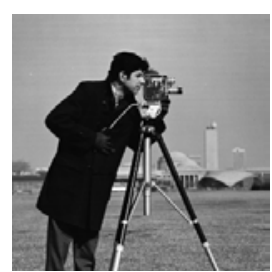

(e)

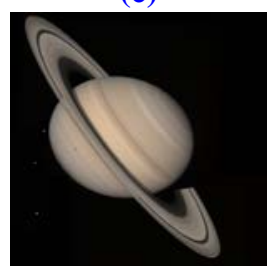

(j)

Fig. 4. Test images. (a) Boat, (b) Fruits, (c) Liftingbody, (d) Phantom, (e) Cameraman, (f) Concordaerial, (g) Bone1, (h) Bone2, (i) Lighthouse, (j) Saturn

Tables 3 and 4 show the reconstruction PSNR of various test images with block sizes $32 \times 16$ and $32 \times 32$, respectively.

Table 3. The reconstruction PSNR (dB) of various test images with block size $32 \times 16$

\begin{tabular}{|c|c|c|c|c|c|}
\hline Images & BSFDBM & Gaussian & Bernoulli & Binary & CsPM \\
\hline Boat & 30.49 & 28.40 & 28.67 & 28.58 & 28.70 \\
\hline Fruits & 27.92 & 25.92 & 26.10 & 25.99 & 25.81 \\
\hline Liftingbody & 36.87 & 35.35 & 35.45 & 35.29 & 35.41 \\
\hline Phantom & 28.28 & 25.52 & 25.32 & 26.24 & 25.69 \\
\hline Cameraman & 25.55 & 23.91 & 24.16 & 23.80 & 24.24 \\
\hline Concordaerial & 30.84 & 29.14 & 29.22 & 29.20 & 29.19 \\
\hline Bone1 & 35.26 & 33.76 & 33.45 & 32.90 & 33.65 \\
\hline Bone2 & 28.79 & 27.70 & 27.55 & 27.43 & 27.63 \\
\hline Lighthouse & 28.39 & 26.96 & 26.75 & 26.97 & 27.07 \\
\hline Saturn & 50.67 & 49.29 & 49.27 & 49.27 & 49.32 \\
\hline
\end{tabular}

Table 4. The reconstruction PSNR (dB) of various test images with block size $32 \times 32$

\begin{tabular}{|c|c|c|c|c|c|}
\hline Images & BSFDBM & Gaussian & Bernoulli & Binary & CsPM \\
\hline Boat & 31.14 & 29.25 & 29.31 & 29.34 & 29.34 \\
\hline Fruits & 28.78 & 26.87 & 26.94 & 26.92 & 26.88 \\
\hline Liftingbody & 37.48 & 36.10 & 36.09 & 35.95 & 36.08 \\
\hline Phantom & 32.00 & 29.95 & 29.69 & 29.76 & 30.16 \\
\hline Cameraman & 26.09 & 24.44 & 24.65 & 24.28 & 24.67 \\
\hline Concordaerial & 31.18 & 29.56 & 29.56 & 29.53 & 29.54 \\
\hline Bone1 & 38.17 & 36.17 & 36.71 & 36.63 & 36.67 \\
\hline
\end{tabular}




\begin{tabular}{|c|c|c|c|c|c|}
\hline Bone2 & 29.53 & 28.22 & 28.17 & 28.11 & 28.29 \\
\hline Lighthouse & 29.02 & 27.59 & 27.43 & 27.55 & 27.44 \\
\hline Saturn & 51.18 & 49.94 & 49.91 & 49.89 & 49.92 \\
\hline
\end{tabular}

From Tables 3 and 4, it can be seen that for all grayscale and color images, the BSFDBM matrix has the highest reconstruction PSNR among the five matrices. Moreover, the reconstruction PSNR increases with the block size increasing.

In the following, we further show the visual results via the "bone2" image reconstruction. Fig. 5 shows the reconstructions with block size $32 \times 16$.

From Fig. 5, it can be seen that the BSFDBM matrix provides competitive visualization performance when compared to the Gaussian, Bernoulli, binary and CsPM matrices.

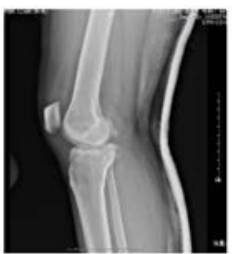

(a)

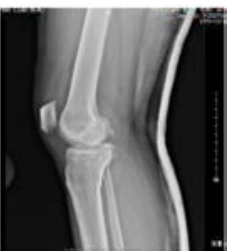

(b)

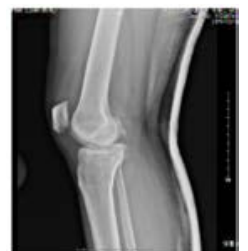

(c)

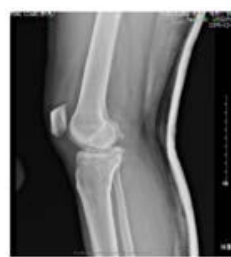

(d)

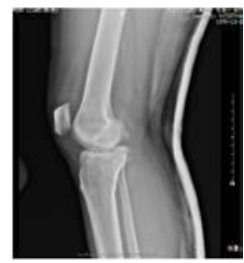

(e)

Fig. 5. Reconstructions with block size 32x16. (a) BSFDBM, (b) Gaussian, (c) Bernoulli, (d) binary, (e) CsPM

\subsection{Computational Complexity of BSFDBM}

Table 2 shows that the multiplier-less operation is supported by BSFDBM, Bernoulli, binary and CsPM matrices, and that the Gaussian matrix doesn't support multiplier-less operation. To compare the computational complexity of the five matrices, we record the reconstruction time, which can characterize the computational complexity. Table 5 shows the reconstruction time in seconds for these images in Fig. 4 with block size $32 \times 16$.

Table 5. The reconstruction time (second) of various test images with block size $32 \times 16$

\begin{tabular}{|c|c|c|c|c|c|}
\hline Images & BSFDBM & Gaussian & Bernoulli & Binary & CsPM \\
\hline Boat & 28.66 & 29.91 & 27.81 & 28.34 & 28.88 \\
\hline Fruits & 27.73 & 29.91 & 28.02 & 28.19 & 27.73 \\
\hline Liftingbody & 112.67 & 118.27 & 111.72 & 113.16 & 111.13 \\
\hline Phantom & 24.23 & 25.72 & 24.72 & 24.58 & 24.98 \\
\hline Cameraman & 30.98 & 33.05 & 31.56 & 31.00 & 30.98 \\
\hline Concordaerial & 7927.34 & 8536.52 & 8014.30 & 8048.41 & 8021.94 \\
\hline Bone1 & 1246.98 & 1353.36 & 1260.72 & 1271.75 & 1266.14 \\
\hline Bone2 & 535.95 & 574.73 & 535.02 & 539.13 & 538.41 \\
\hline Lighthouse & 487.41 & 527.00 & 493.86 & 493.88 & 491.27 \\
\hline Saturn & 2343.08 & 2527.25 & 2370.86 & 2380.83 & 2365.53 \\
\hline
\end{tabular}


Table 5 shows that for all these matrices, the Gaussian matrix has the longest reconstruction time. This is because that the additional multiplication operation introduces more reconstruction time. Numerical results show that the BSFDBM can reduce the computational complexity compared with its Gaussian counterpart. Particularly, the complexity gain is large when reconstructing color images "concordaerial", "bone1" and "Saturn". This corresponds to a large-scale data scenario. In addition, there is not much difference of reconstruction time among the BSFDBM, Bernoulli, binary and CsPM matrices.

Numerical simulations with sparse signals and images show that the reconstruction accuracy of BSFDBM matrices is superior to the Gaussian, Bernoulli, binary and CsPM matrices, which coincides with the conclusion of Theorem 4.2 and Corollary 4.1. The BSFDBM matrices can also reduce the reconstruction time compared with their Gaussian counterpart. Consequently, the designed BSFDBM matrices inspired from BSF possess the practical features of good sensing performance, low memory cost, low computational complexity and easy hardware implementation. These features can make the proposed BSFDBM matrices applied to practical scenarios of CS application, including sparse signal restore and image block CS.

\section{Conclusion}

Based on BSF, this paper proposes a novel method of constructing bipolar measurement matrices named BSFDBM and gives related LFSR implementation. For BSFDBM matrices, the coherence is given in different situations and proved to be smaller than that of corresponding Gaussian and Bernoulli matrices via theoretical derivation. Moreover, the corresponding practical features of BSFDBM are analyzed and compared. Simulation experiments show that the BSFDBM matrices outperform their Gaussian, Bernoulli, binary and chaotic bipolar counterparts in reconstruction accuracy. The BSFDBM matrices can also reduce the computational complexity compared with their Gaussian counterpart. The BSFDBM matrices are very efficient for sensing performance, memory, complexity and hardware realization, which is beneficial to practical CS.

\section{References}

[1] Emmanuel J. Candès, Justin Romberg and Terence Tao, "Robust uncertainty principles: exact signal reconstruction from highly incomplete frequency information," IEEE Transactions on Information Theory, vol. 52, no. 2, pp. 489-509, February, 2006. Article (CrossRef Link).

[2] David L. Donoho, “Compressed sensing,” IEEE Transactions on Information Theory, vol. 52, no. 4, pp. 1289-1306, April, 2006. Article (CrossRef Link).

[3] Emmanuel J. Candès and Terence Tao, “Decoding by linear programming,” IEEE Transactions on Information Theory, vol. 51, no. 12, pp. 4203-4215, December, 2005. Article (CrossRef Link).

[4] Jean Bourgain, Stephen Dilworth, Kevin Ford, Sergei Konyagin and Denka Kutzarova, "Explicit constructions of RIP matrices and related problems,” Duke Mathematical Journal, vol. 159, no. 1, pp. 145-185, 2011. Article (CrossRef Link).

[5] Hongping Gan, Zhi Li, Jian Li, Xi Wang and Zhengfu Cheng, "Compressive sensing using chaotic sequence based on chebyshev map,” Nonlinear Dynamics, vol. 78, no. 4, pp. 2429-2438, December, 2014. Article (CrossRef Link).

[6] Juan Castorena and Charles D. Creusere, "The restricted isometry property for banded random matrices,” IEEE Transactions on Signal Processing, vol. 62, no. 19, pp. 5073-5084, October, 2014. Article (CrossRef Link). 
[7] Hongping Gan, Song Xiao and Yimin Zhao, “A novel secure data transmission scheme using chaotic compressed sensing,” IEEE Access, vol. 6, pp. 4587-4598, February, 2018. Article (CrossRef Link).

[8] Mahsa Lotfi and Mathukumalli Vidyasagar, "A fast noniterative algorithm for compressive sensing using binary measurement matrices,” IEEE Transactions on Signal Processing, vol. 66, no. 15, pp. 4079-4089, May, 2018. Article (CrossRef Link).

[9] Hongping Gan, Song Xiao, Tao Zhang and Feng Liu, "Bipolar measurement matrix using chaotic sequence,” Communications in Nonlinear Science and Numerical Simulation, vol. 72, pp. 139-151, June, 2019. Article (CrossRef Link).

[10] Shuxing Li and Gennian Ge, "Deterministic sensing matrices arising from near orthogonal systems,” IEEE Transactions on Information Theory, vol. 60, no. 4, pp. 2291-2302, April, 2014. Article (CrossRef Link).

[11] Li Zeng, Xiongwei Zhang, Liang Chen, Tieyong Cao and Jibin Yang, "Deterministic construction of toeplitzed structurally chaotic matrix for compressed sensing," Circuits, Systems, and Signal Processing, vol. 34, no. 3, pp. 797-813, March, 2015. Article (CrossRef Link).

[12] Jun Zhang, Guojun Han and Yi Fang, "Deterministic construction of compressed sensing matrices from protograph LDPC codes,” IEEE Signal Processing Letters, vol. 22, no. 11, pp. 1960-1964, November, 2015. Article (CrossRef Link).

[13] Guohua Zhang, Rudolf Mathar and Quan Zhou, "Deterministic bipolar measurement matrices with flexible sizes from Legendre sequence,” Electronics Letters, vol. 52, no. 11, pp. 928-930, May, 2016. Article (CrossRef Link).

[14] Tian Shujuan, Fan Xiaoping, Li Zhetao, Pan Tian, Choi Youngjune and Sekiya Hiroo, "Orthogonal-gradient measurement matrix construction algorithm," Chinese Journal of Electronics, vol. 25, no. 1, pp. 81-87, January, 2016. Article (CrossRef Link).

[15] R. Ramu Naidu, Phanindra Jampana and C. S. Sastry, "Deterministic compressed sensing matrices: construction via Euler Squares and applications,” IEEE Transactions on Signal Processing, vol. 64, no. 14, pp. 3566-3575, July, 2016. Article (CrossRef Link).

[16] Pradip Sasmal, R. Ramu Naidu, Challa S. Sastry and Phanindra Jampana, "Composition of binary compressed sensing matrices,” IEEE Signal Processing Letters, vol. 23, no.8, pp. 1096-1100, August, 2016. Article (CrossRef Link).

[17] R. Ramu Naidu and Chandra R. Murthy, "Construction of binary sensing matrices using extremal set theory,” IEEE Signal Processing Letters, vol. 24, no. 2, pp. 211-215, February, 2017. Article (CrossRef Link).

[18] Weizhi Lu, Tao Dai and Shu-Tao Xia, "Binary matrices for compressed sensing," IEEE Transactions on Signal Processing, vol. 66, no. 1, pp. 77-85, January, 2018.

Article (CrossRef Link).

[19] Hongping Gan, Song Xiao, Yimin Zhao and Xiao Xue, "Construction of efficient and structural chaotic sensing matrix for compressive sensing," Signal Processing: Image Communication, vol. 68, pp. 129-137, October, 2018. Article (CrossRef Link).

[20] Hongping Gan, Song Xiao and Yimin Zhao, "A large class of chaotic sensing matrices for compressed sensing,” Signal Processing, vol. 149, pp. 193-203, August, 2018. Article (CrossRef Link).

[21] Liu Haiqiang, Yin Jihang, Hua Gang, Yin Hongsheng and Zhu Aichun, "Deterministic construction of measurement matrices based on Bose balanced incomplete block designs," IEEE Access, vol. 6, pp. 21710-21718, April, 2018. Article (CrossRef Link).

[22] Gang Wang, Min-Yao Niu and Fang-Wei Fu, "Deterministic constructions of compressed sensing matrices based on optimal codebooks and codes,” Applied Mathematics and Computation, vol. 343, pp. 128-136, February, 2019. Article (CrossRef Link).

[23] Haiyang Liu, Hao Zhang and Lianrong Ma, "On the spark of binary LDPC measurement matrices from complete protographs,” IEEE Signal Processing Letters, vol. 24, no. 11, pp. 1616-1620, November, 2017. Article (CrossRef Link). 
[24] Jue Wang, Zhaoyang Zhang, Xianbin Wang, Hong Wang and Chunxu Jiao, "A low-complexity reconstruction algorithm for compressed sensing using Reed-Muller sequences," in Proc. of IEEE Int. Conf. on Communications, pp. 1-6, May 20-24, 2018. Article (CrossRef Link).

[25] Sung-Hsien Hsieh, Chun-Shien Lu and Soo-Chang Pei, "Compressive sensing matrix design for fast encoding and decoding via sparse FFT," IEEE Signal Processing Letters, vol. 25, no. 4, pp. 591-595, April, 2018. Article (CrossRef Link).

[26] Mohammad Fardad, Sayed Masoud Sayedi and Ehsan Yazdian, "A low-complexity hardware for deterministic compressive sensing reconstruction," IEEE Transactions on Circuits and Systems I: Regular Papers, vol. 65, no. 10, pp. 3349-3361, October, 2018. Article (CrossRef Link).

[27] Nam Yul Yu and Guang Gong, "A new binary sequence family with low correlation and large size," IEEE Transactions on Information Theory, vol. 52, no. 4, pp. 1624-1636, April, 2006. Article (CrossRef Link).

[28] Joel A. Tropp, "Greed is good: Algorithmic results for sparse approximation," IEEE Transactions on Information Theory, vol. 50, no. 10, pp. 2231-2242, October, 2004. Article (CrossRef Link).

[29] Scott Shaobing Chen, David L. Donoho and Michael A. Saunders, "Atomic decomposition by basis pursuit,” SIAM Journal on Scientific Computing, vol. 20, no. 1, pp. 33-61, August, 1998. Article (CrossRef Link).

[30] David L. Donoho and Michael Elad, "Optimally sparse representation in general (nonorthogonal) dictionaries via $l 1$ minimization," Proceedings of the National Academy of Sciences, vol. 100, no. 5, pp. 2197-2202, March, 2003. Article (CrossRef Link).

[31] Jarvis Haupt, Waheed U. Bajwa, Gil Raz and Robert Nowak, "Toeplitz compressed sensing matrices with applications to sparse channel estimation," IEEE Transactions on Information Theory, vol. 56, no. 11, pp. 5862-5875, November, 2010. Article (CrossRef Link).

[32] Robert Gold, "Maximal recursive sequences with 3-valued recursive cross-correlation functions," IEEE Transactions on Information Theory, vol. 14, no. 1, pp. 154-156, January, 1968. Article (CrossRef Link).

[33] Udaya Parampalli, "Polyphase and frequency hopping sequences obtained from finite rings," Ph.D. dissertation, Department of Electrical Engineering, Indian Institute of Technology, Kanpur, India, 1992.

[34] Oscar S. Rothaus, "Modified Gold codes," IEEE Transactions on Information Theory, vol. 39, no. 2, pp. 654-656, March, 1993. Article (CrossRef Link).

[35] Abhijit. G. Shanbhag, P. Vijay Kumar and Tor Helleseth, "Improved binary codes and sequence families from $Z_{4}$-linear codes," IEEE Transactions on Information Theory, vol. 42, no. 5, pp. 1582-1587, September, 1996. Article (CrossRef Link). 


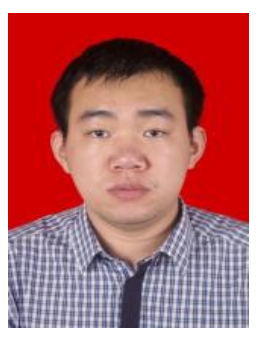

Cunbo Lu is currently a postdoctoral researcher of Beijing Institute of Applied Physics and Computational Mathematics. He received his Bachelor degree from PLA Information Engineering University, Zhengzhou, China and his Ph.D. degree from Xidian University, Xi'an, China. His research interests include network coding, wireless network, image processing and compressed sensing.

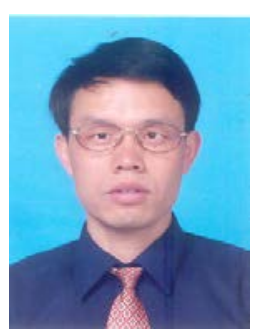

Wengu Chen received the Ph.D. degree from Beijing Normal University in 1996 in mathematics. He is currently a professor of Beijing Institute of Applied Physics and Computational Mathematics. His research interests include harmonic analysis, channel coding, and compressive sensing.

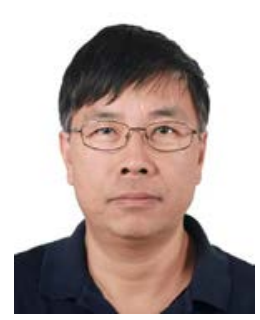

Haibo Xu received the Ph.D. degree from Graduate School of China Academy of Engineering physics. He is currently a professor of Beijing Institute of Applied Physics and Computational Mathematics. His research interests include radiation imaging and image processing. 